\title{
Study on Computer Model Algorithm of Laying Strategy in Garment Layout Technology Li-na Cui
}

\author{
Deparment of Arts and Design, Quanzhou Normal University \\ Quanzhou, P.R. China; \\ e-mail: cuilina-cui@163.com
}

Keywords: component; Garment layout; mathematical expression; laying strategy; computer algorithm

\begin{abstract}
The key problem of garment layout is laying as much garment patterns as possible in the certain 2D fabric, making sure the fabric's using rate high enough as far as possible. This study takes mathematical analysis and computer algorithms research to garment layout strategy problem whose target is computer intelligentialize.
\end{abstract}

\section{INTRODUCTION}

Layout is a typical problem of optimization and combination, With the rapid development of computer technology and the wide application of computer aided design, If combining the traditional artificial layout experience and artificial intelligence technology, the characteristics of computer's huge capacity, fast operation speed will take effect, under the premise of achieving the desired material utilization rate, the rate is far higher than the artificial layout.

\section{THE ESTABLISHMENT OF MATHEMATICAL MODEL OF CLOTHING LAYOUT PROBLEM}

In order to solving the clothing of two-dimensional layout optimization problem, first of all, the problem of the reasonable laying the garment patterns ready to be laid so as to save material should be described with appropriate mathematical model, and the variables have to be determined, the objective function and constraint conditions have to be listed on. Because of the garment patterns are rigid, so the position fixing of the garment patterns ready to be laid in the fabric actually need only 3 parameters. These 3 parameters are the coordinates of the reference points in the pattern on the fabric $(\mathrm{x}, \mathrm{y})$ and the pattern's display angle. When determining these 3 parameters, the absolute coordinates of the pattern can be calculated by these 3 parameters. Centre of gravity is taken as the reference point of the pattern generally. Take the fabric width, length direction as the $\mathrm{X}, \mathrm{Y}$ axis, and the lower left corner of the fabric as the origin point to establish the plane coordinate system [1]. The orientation of each pattern in the fabric can be represented as such a process: firstly the pattern rotates by $\theta$ degree around the centre of gravity and makes the fabric of the minimum width of the bounding rectangle by the warp direction. After calculation of extrusion algorithm, the pattern's reference point in the fabric ( $\mathrm{x} 0, \mathrm{y} 0)$ can be concluded, in the extrusion process the layout angle $\theta$ will not be changed. Then the pattern i's position in fabric can be expressed as $\mathrm{Gi}(\mathrm{x}, \mathrm{y}, \theta)$, let $\mathrm{Si}$ be the area of pattern i, the fabric's width $\mathrm{W}$ and length $\mathrm{L}$ is unfixed, the materials' utilization rate is Emax, if the value is greater and then the using rate of fabrics is higher, then the clothing layout optimization model is as follow:

$$
\sum_{i=1}^{n} s_{i} /(W * L) \quad i=1,2,3 \ldots \ldots n
$$

The constraint conditions are as follow:

$$
\begin{aligned}
& \mathrm{G}_{\mathrm{i}}\left(\mathrm{x}_{\mathrm{i}}, \mathrm{y}_{\mathrm{i}}, \theta_{\mathrm{i}}\right) \cap \mathrm{G}_{\mathrm{j}}\left(\mathrm{x}_{\mathrm{j}}, \mathrm{y}_{\mathrm{j}}, \theta_{\mathrm{j}}\right)=\Phi \\
& 0 \leqq \mathrm{x}_{\mathrm{i}}, \mathrm{x}_{\mathrm{j}} \leqq \mathrm{W}, 0 \leqq \mathrm{y}_{\mathrm{i}}, \mathrm{y}_{\mathrm{j}} \leqq \mathrm{L}
\end{aligned}
$$

Constraint condition (2) shows the pattern $\mathrm{i}$ and pattern j do not overlap each other; constraint condition (3) shows the patterns cannot be laid outside of the fabric region. 


\section{The MATHEMATICAL EXPRESSION METHOD OF GARMENT LAYOUT PROBLEM}

A. The Mathematical Expression of the Pattern Ready to be Laid

Inspired by the scanning technique of raster graphics, two-dimensional irregular polygons can be represented by the intersection area of horizontal scan lines with equal distance and the polygon. The endpoints of the area can be obtained through the intersection point of scan lines and polygon boundary line. As shown in Figure 1, intersection points of polygon scan line 2( line $y=2$ )and polygon are $\mathrm{Pl}(2,2)$ and $\mathrm{E}(8,2)$, so the scanning area of the polygon scan line 2 is $[2,8]$; intersection points of polygon scan line 6 ( line $y=6$ )and polygon are A $(2,6), B(3.5,6), C(7,6), D$ $(11,6)$, so the scanning area of the polygon scan line 6 is $[2,3.5]$ and $[7,11]$.

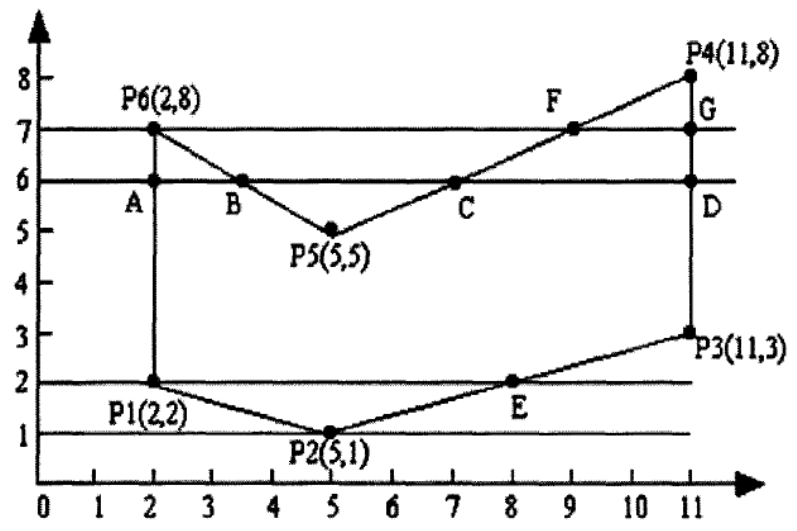

Figure 1. polygon horizontal scan lines and boundary line

For the scan line, polygon scan conversion process can be divided into five steps:

1) Seek the intersection: calculate the intersection point of scanning line and the edges of polygon;

2) Accept and reject points: if the intersection points of scanning line and the edges of polygon locate on two sides of the scanning line respectively, we take one point into consideration. In Figure $1, \mathrm{P}_{5}$ and $\mathrm{P}_{6}$ scan lines and polygon intersection edges are respectively positioned in the same side scan lines, and the $y \mathrm{i}<\mathrm{yi}-1, \mathrm{yi}<\mathrm{yi}+1$, we take 2 points into consideration, such asP2; If $\mathrm{yi}>\mathrm{yi}-1, \mathrm{yi}>\mathrm{yi}+1$, and 0 point is considered, such as $\mathrm{P} 1$ in Figure 1 ; If the scanning line coincide with the polygon's boundary, then we consider1 intersection point.

3) Ranking: sort all the intersection points by the $x$ value's increasing order;

4) Matched: the first and second, third and fourth and so on; each pair of intersection point represents the intersection area of scanning line and the polygon.

5) Record intersection area.

B. Geometry Calculation of Garment Patterns ready to be laid

In garment layout process, the corresponding polygon of garment pattern will be put to various geometric operations according to need, such as polygon moving ,polygon area calculation, polygon rotating ,and polygon reflection, etc. The geometric calculation method is introduced in this paper as following.

1) Polygon's area calculation

Polygon's area calculation result is mainly used in the calculation of the material utilization rate, and we take the result as the evaluation standard of layout scheme. Because the polygon is irregular in shape, so we cannot simply calculate the area of a polygon. However, we can use polygon segmentation algorithm, the irregular polygon can be divided into triangular, rectangular and trapezoidal combined simple graphics, then calculate these simple graphics area respectively and add them up. Given irregular polygon $\mathrm{P}$ consists of $\mathrm{n}$ endpoints, all endpoints are connected in sequence:

$\mathrm{P}=\left\{\mathrm{P}_{1}\left(\mathrm{x}_{1}, \mathrm{y}_{1}\right), \mathrm{P}_{2}\left(\mathrm{x}_{2}, \mathrm{y}_{2}\right), \ldots, \mathrm{P}_{\mathrm{n}}\left(\mathrm{x}_{\mathrm{n}}, \mathrm{y}_{\mathrm{n}}\right)\right\}$, so we can derive irregular polygon's area formula as follow:

$$
\mathrm{S}=\frac{1}{2} \sum_{i=1}^{n} \mathrm{x}_{\mathrm{i}} \mathrm{y}_{\mathrm{i}+1}-\mathrm{x}_{\mathrm{i}+1} \mathrm{y}_{\mathrm{i}}
$$

2) The polygon's movement

Polygon's moving is mainly used to lay the polygon on some position during layout process, 
and after the operation of being rotated, mirrored, the polygon can be moved back to the first quadrant. Take the minimum value in the $\mathrm{X}$ coordinate, the minimum value in $\mathrm{Y}$ coordinates as the basis point, and then translate the polygon horizontal and vertical some distance to the anchor point.

3) The polygon's rotation

Polygon rotates around its center of gravity $G\left(x_{g}, y_{g}\right), x_{g}=\sum_{i=1}^{n} x_{i} / n, y_{g}=\sum_{i=1}^{n} y_{i} / n$, The transformation formula for polygon rotates around its center of gravity is as follows[2]:

$\mathrm{x}^{\prime}=\mathrm{x} \cos \theta-\mathrm{y} \sin \theta+(1-\cos \theta) \mathrm{x}_{\mathrm{g}}+\mathrm{y}_{\mathrm{g}} \sin \theta$

$\mathrm{y}^{\prime}=\mathrm{x} \sin \theta-\mathrm{y} \cos \theta+(1-\cos \theta) \mathrm{y}_{\mathrm{g}}+\mathrm{x}_{\mathrm{g}} \sin \theta$

$C$. Close Together for the Garment Patterns ready to be laid

Suppose we want to determine Whether a point $\operatorname{pos}(\mathrm{x}, \mathrm{y})$ on the fabric can lay the pattern, for each corresponding scan interval [px1, px2] of pattern scan line and each corresponding scan interval [sx1, sx2] of fabric scan line, if the flag position of fabric scanning interval [sx1, sx2] dirtyFlag=true, and [px1, px2] and [sx1, sx2] are overlapping, then the current pattern cannot be laid on point POS $(\mathrm{x}, \mathrm{y})$, move the pattern to right for a distance dis= sx2-px1, at the same time positioning point also move the same distance dis=sx2-px1, go on to judge, until the pattern ready to be laid are completed or give up.

\section{COMPUTER MODEL ALGORITHM OF LAYING STRATEGY IN GARMENT LAYOUT TECHNOLOGY}

How to lay the patterns to the fabric reasonably and orderly under the condition of fabric utilization ratio is relatively high, that is the strategy problem of layout. In 1980, Bake put forward an algorithm based on BL (Bottom-Left) heuristic localization algorithm.

The BL algorithm is a layout algorithm under the principle of meeting "far left and far bottom". That is to say, in the layout process, the patterns under the case of not being overlap with each other or beyond the scope of the fabric, each pattern move as far as possible to the left and bottom, until it can no longer move any more [3]. The method is simple and easy, but in the layout process, after the larger pattern finishing laying ,it may stop moving subsequent patterns, resulting in small patterns could not be filled to the blank area of the bottom and left.

Thus, researchers have proposed the improved BLF (Bottom-Left-Fill) algorithm, which can fill the blank area formed in the layout process in fabric, improve the utilization rate of the fabric. The idea of BLF algorithm is starting from the leftmost and bottommost position each time, checking whether the pattern ready to be laid can be laid in the blank area of the fabric. This algorithm makes full use of the fabric's blank area in layout process, and compared with the heuristic localization algorithm based on BL strategy, the utilization rate of fabric is obviously higher $[4,5]$.

This paper uses the BLF algorithm and realizes the BLF layout in the fabric. The algorithm process is as follows:

A. Take the pattern's rectangular closure's lower left corner point as the starting point, move the pattern to lower left corner of the fabric's rectangular closure POS (x,y). Pay attention to the moving of the pattern, its corresponding scan line, and all the scan line's scan interval will have to move also;

B. Use fabric's layout position POS (x, y) judgment algorithm, judge whether the current pattern can be laid on the position POS (x,y): if ok, then lay the current pattern on POS (x,y), then begin to continue to move next pattern from step 1;

C. If current pattern cannot be laid on the POS (x,y), then judge from fabric's position POS (x, y) and obtain horizontal displacement "dis" in X axis direction in the algorithm. And then move the current pattern for horizontal displacement "dis" in X axis direction:

If the current pattern being laid beyond the scope of the rectangular fabric closure in $\mathrm{X}$ axis direction, then $\mathrm{y}=\mathrm{y}+1, \mathrm{x}=0$;

If the current pattern being laid beyond the scope of the rectangular fabric closure in $\mathrm{Y}$ axis direction, then abandon current pattern;

Turn to (1), go on move the next pattern;

Turn to (2); 
D. Finishing all patterns, and exit the algorithm.

Among them, the algorithm used to judge whether the position POS (x, y) on the fabric can lay the current pattern is as follow:

1) From the heuristic algorithm based on the BLF, we can obtain position POS (x, y) and the pattern ready to be laid. Initialize the integer variable $\mathrm{i}=0$;

2) For the current pattern, adopt all scan interval in scan line i; for fabrics, adopt all scan interval in the $y+i$ scan line;

3) For each scan interval [px1, px2] of the current pattern's scan line i, and for each scan interval [sx1, sx2] of the fabric's scan line $y+i$, if the flag position of the fabric's scanning interval [sx1, $\mathrm{sx} 2]$ dirtyFlag=true, and the [px1, px2] and [[sx1, sx2] is overlapping, then the pattern cannot be laid in current position POS $(x, y)$, calculate the shift distance dis= sx2-px1 the pattern need to, return "false", return to the shift distance "dis" needed, exit the algorithm;

4) (4) $\mathrm{i}=\mathrm{i}+\mathrm{l}$;

If $\mathrm{i}>\mathrm{N}$, " $\mathrm{N}$ " is the total number of scan lines which go through current pattern, then the current pattern can be laid in the POS (x, y), return true, exit the algorithm. Otherwise, turn to (2).

\section{CONCLUSION}

Apparel 2D irregular layout problem refers to the intelligent optimization algorithms, computational geometry, computer graphics and other theories, this paper introduces the geometry expression method by horizontal line scan interval calculation algorithm and irregular polygon's area calculation, rotating, mobile and other geometry algorithm, furthermore, the layout strategy problem is also investigated, using heuristic methods that based on BLF and horizontal scan lines interval expression, and gives the steps used to judge the overall fabric whether a position can layout the pattern ready to be laid.

\section{References}

[1] Y.Chen. "Study on layout of two dimensional irregular shape's optimization" HangZhou: ZheJiang University.2003;

[2] H.Hu.”An algorithm for rotating polygons contractions", Research and development of computer. 1998, 35(6):567-571

[3] J.M Liu, S.N Zhang. "Research and implementation of algorithm of two dimensional irregular shape material automatic layout”, Journal of Computer Aided Design \& Computer Graphics. 2000, 12(7):458-491

[4] X.X Liu, C.Y Xu. “Automatic layout design of irregular graphics sample”. Journal of Huazhong University of Science and Technology (NATURAL SCIENCE EDITION). 2002, 30(2):19-20

[5] CY. Huang. X.Zhang, Analysis on neck clustering of young men and size specifications of shirts[J] : Journal of Textile Research, 2007(1):91-93 